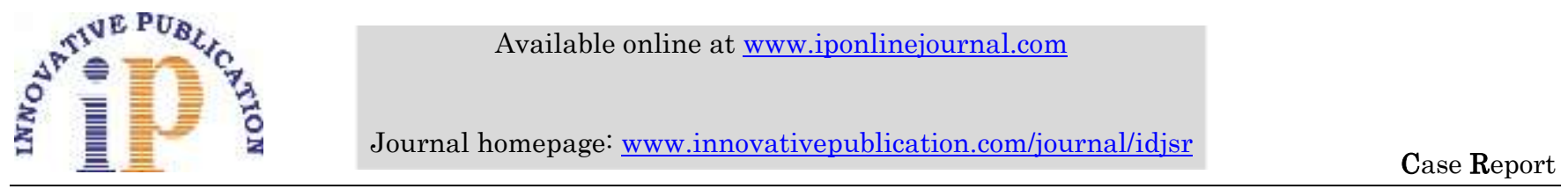

\title{
A Scope for Advancement
}

\author{
Binu Purushothaman', Nevin Abraham², Anju Ramachandran ${ }^{3 *}$, Pooja Sanal ${ }^{4}$, Shoma Renjith $^{5}$ \\ ${ }^{1}$ Professor and HOD, ${ }^{2-5}$ Post Graduate Student, Dept. of Orthodontics, ${ }^{1-5}$ KMCT Dental College, Kozhikode, Kerala, India
}

\begin{abstract}
Skeletal Class II malocclusion may be due to maxillary prognathism, mandibular retrognathism or a combination of both. Among these, mandibular retrusion is one of the most common characteristics in Skeletal Class II patients.

Early screening and diagnosis help in preventing and intercepting the severity of the malocclusion. Mostly, Class II Skeletal discrepancies with mandibular deficiency during active growth are usually treated by myofunctional appliances. Recently, fixed functional appliance therapy has been gaining popularity as a treatment modality of skeletal class II malocclusion.

This case report demonstrates the effectiveness of Power Scope in correction of skeletal Class II with mandibular deficiency in a growing patient treated by a non-extraction approach. This case was treated initially with MBT 0.022 ” prescription followed by Power Scope Appliance. Results were improvement in facial profile, skeletal jaw relationship, and overall esthetic appearance of the patient.
\end{abstract}

Keywords: Class II malocclusion, Non-Extraction, Power scope appliance.

\section{Introduction}

According to McNamara, the most common characteristic of Class II malocclusion is mandibular retrusion, rather than maxillary prognathism. ${ }^{3}$

A functional appliance treatment for mandibular advancement is often advocated in Class II malocclusion due to mandibular retrusion. Fixed functional appliance therapy has recently been gaining immense popularity as a means to noncompliant correction of skeletal class II malocclusion. ${ }^{4}$

Power Scope (Fig. 1) is the latest innovation in Class II correction which is a direct derivative of the Herbst Type II appliance. $^{1,2}$ Dr. Andy Hayes worked in conjunction with American Orthodontics to develop Power Scope. Its unique features are patient-friendly design, ready to use one piece with no laboratory setup and no assembly. It has a simple attachment system with durable telescopic mechanism, a $\mathrm{Ni}-\mathrm{Ti}$ internal spring system which reduces the treatment time compared to the other Class II correctors and a ball and socket joint system which maximizes lateral movement for patient comfort.

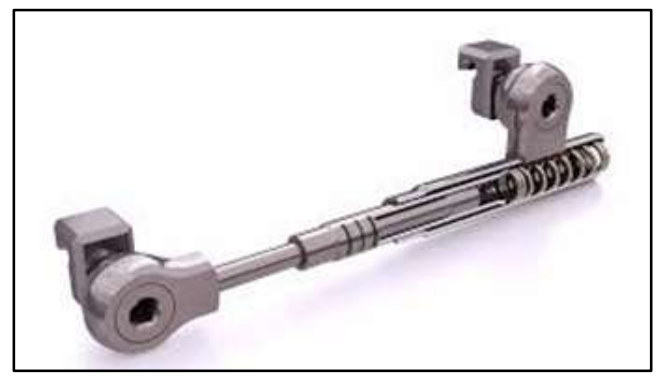

Fig 1: POWERSCOPE

\section{Case Report}

A 14-year-old female patient came to the department of orthodontics, KMCT (Calicut) Kerala with chief complaint of forwardly placed upper front teeth.

Extra oral examination (Fig. 2) revealed a slight convex profile with posterior divergence, and lip incompetency.
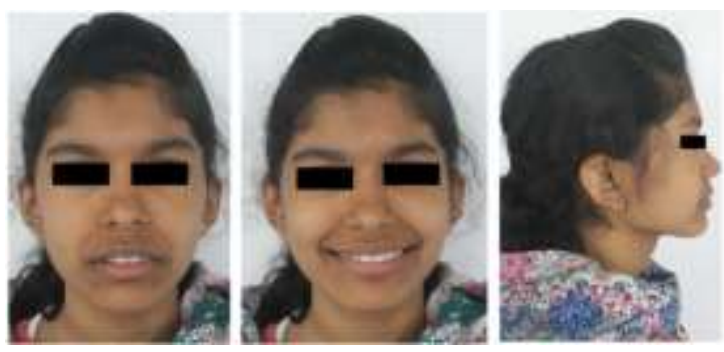

Fig. 2: Extra oral photographs

Intraoral examination (Fig. 3) revealed a Class II, Division 1 malocclusion with an overjet of $8 \mathrm{~mm}$ and an overbite of $5 \mathrm{~mm}$, with a coinciding upper and lower dental and skeletal midline.

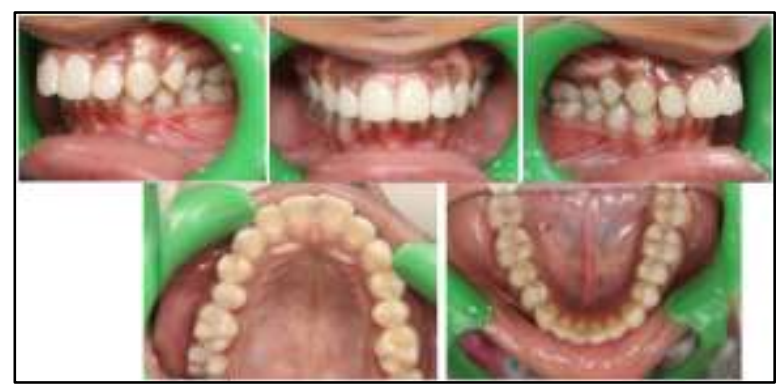

Fig. 3: Intraoral photographs

\footnotetext{
*Corresponding Author: Anju Ramachandran, Post Graduate Student, Dept. of Orthodontics, KMCT Dental College, Kozhikode, Kerala, India

Email: dranjurchandran@gmail.com

http://doi.org/10.18231/j.idjsr.2019.016
} 
Cephalometric analysis (Fig. 4) revealed a convex skeletal profile with ANB angle of $5^{\circ}$, a retruded mandible, and a well-positioned maxilla. Dentoalveolar readings suggested proclined upper anterior teeth while the lower incisors were properly inclined. The panoramic radiograph revealed the presence of all permanent teeth with erupting third molars.
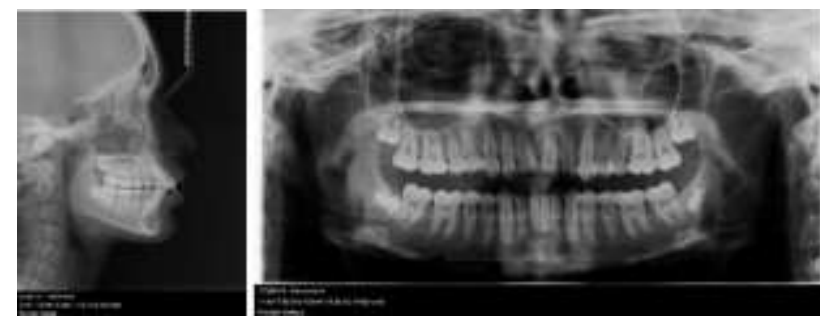

Fig. 4: Pretreatment radiographs

\section{Treatment plan}

Treatment goals were to correct the patient's skeletal relationship and improve the facial profile by accentuating mandibular growth and to attain a Class I molar relation on either side.

A nonextraction approach was planned using MBT 0.022 " slot preadjusted appliance.

Treatment was started using 0.014 " NiTi in both arches followed by 0.017 "X 0.025 " NiTi and 0.017 "X 0.025 " SS. Leveling and alignment was completed in 5-months time period.

After leveling and aligning,.019" $\times .025 "$ stainless steel arch wires were inserted. TPA was given in upper arch.

Power Scope (Fig. 5) was placed between the upper and lower arch for placing the mandible forward to improve patient's profile. Because it is worn full-time, it does not depend on patient cooperation. Fixed functional therapy was completed with a duration of 6 months. Entire treatment time was 1.5 years
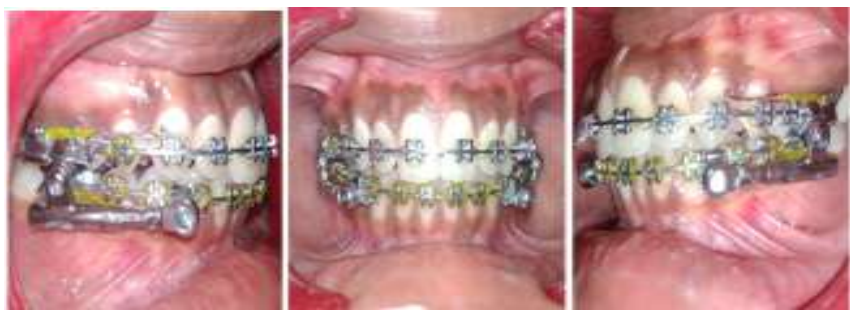

Fig. 5: With power scope

\section{Results}

Results was pleasing for the patient. Profile (Fig. 6) was improved by the forward placement of mandible after the treatment. Patient attained a Class I molar relation, ideal overjet and overbite.
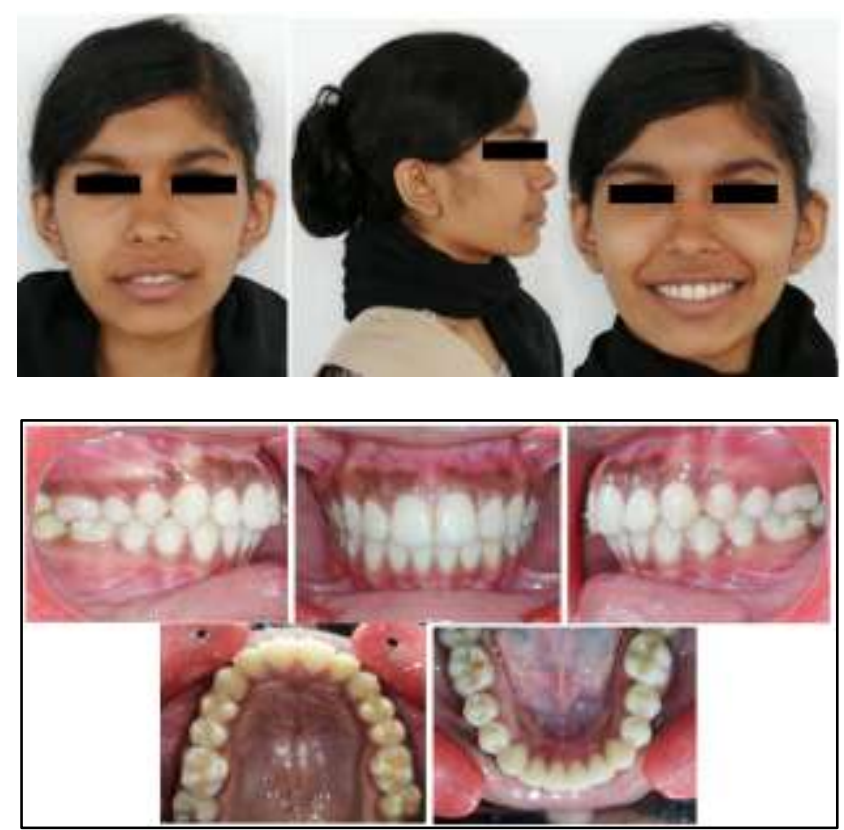

Fig. 6: Post treatment extra oral and intra oral photographs

The posttreatment cephalometric measurements (Fig. 7) revealed favorable sagittal skeletal changes. A mandibular advancement was clearly evident as ANB angle was reduced attaining a Class I skeletal profile.
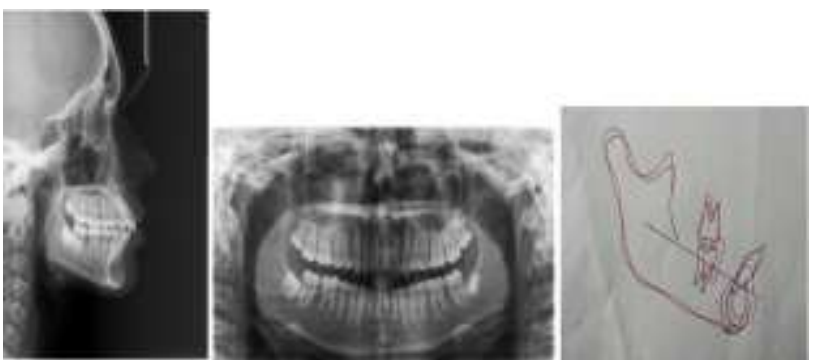

Fig.7: Post treatment radiographs and superimposition

Cephalometric comparison revealed a drastic improvement in patient's profile along with an increase in the airway space which was achieved by forward placement of mandible.

Table 1: shows the comparison of pre and post cephalometric radiographic readings.

\begin{tabular}{|c|c|c|}
\hline & Skeletal Analysis & \\
\hline Parameters & Pretreatment & Post treatment \\
\hline SNA angle & $83^{0}$ & $83^{0}$ \\
\hline SNB angle & $78^{0}$ & $80^{0}$ \\
\hline ANB angle & $5^{0}$ & $3^{0}$ \\
\hline
\end{tabular}




\begin{tabular}{|c|c|c|}
\hline Go-Gn to SN & $23^{0}$ & $28^{0}$ \\
\hline & Dental Analysis & $25^{0}$ \\
\hline U1 -NA angle (degree) & $34^{\circ}$ & $5 \mathrm{~mm}$ \\
\hline U1 -NA (mm) & $8 \mathrm{~mm}$ & $109^{0}$ \\
\hline U1 -SN angle (degree) & $118^{0}$ & $42^{0}$ \\
\hline L1-NB angle (degree) & $40^{0}$ & $6 \mathrm{~mm}$ \\
\hline L1-NB (mm) & $5 \mathrm{~mm}$ & $4 \mathrm{~mm}$ \\
\hline L1-A pog (mm) & $3 \mathrm{~mm}$ & $102^{0}$ \\
\hline IMPA & $100^{0}$ & $110^{0}$ \\
\hline Interincisal angle & $105^{0}$ & $+1 \mathrm{~mm}$ \\
\hline S line-U lip (mm) & Soft Tissue Analysis & $-2 \mathrm{~mm}$ \\
\hline S line -L lip (mm) & $+2 \mathrm{~mm}$ & 109 \\
\hline Nasolabial Angle & $-3 \mathrm{~mm}$ & $90^{0}$ \\
\hline
\end{tabular}

As a part of retention phase, Beggs wrap around retainer along with an anterior inclined bite plane was given in the upper arch. Fixed lingual retainers were bonded in both the arches

\section{Conclusion}

In this case, Power Scope proved to be one of the best treatment modalities for Skeletal Class II correction by utilizing remaining growth potential of patient by sagittal forward displacement of the mandible. Moreover, it offered an improvement in the facial profile in a non-extraction approach ensuring excellent long-term stable results.

\section{Source of Funding}

None.

\section{Conflict of Interest}

None.

\section{References}

1. Paulose J, Antony PJ, Suresh Kumar B, George SM, Mathew MM, Sebastian J. Power Scope a Class II corrector-A case report. Contemp Clin Dent 2016;7(2):221

2. Bohara P. Correction of Class II Division 2 Malocclusion by Fixed Functional Class II Corrector Appliance: Case Report. $J$ Contemp Orthod 2018;2(1):54-60

3. McNamara JA Jr. Components of class II malocclusion in children 8-10 years of age. Angle Orthod 1981;51:177-202.

4. Savanna K, Kumar M, Ansari A, Jain A. Correction of Class II using Power scope Appliance-A case report. Arch Dent and Med Res 2016;2(3):120-5.

How to cite this article: Purushothaman B, Abraham N, A Ramachandran, Sanal P, Renjith S, A Scope for Advancement, Int Dent J Student Res 2019;7(3):65-7. 